\title{
EVALUACIÓN DE LA PREVALENCIA ESTIMADA DE SOBREPESO Y OBESIDAD, EN POBLACIONES DE NIÑOS Y ADOLESCENTES DE LA REGIÓN CHAQUEÑA, CON DOS REFERENCIAS INTERNACIONALES
}

\author{
Assessment of Estimated Prevalence of Overweight and Obesity in Children and \\ Adolescents in the Chaco Region, with Two International References
}

Gisel Padula y Susana A. Salceda

\begin{abstract}
Resumen
La evaluación de la situación nutricional de los niños, depende en gran medida de las curvas de crecimiento utilizadas. El objetivo de esta investigación es comparar las prevalencias de sobrepeso y obesidad estimadas con dos referencias internacionales en una población de niños y adolescentes de la Región Chaqueña, a través del Índice de Masa Corporal. Se incluyeron 299 niños de 6 a 15 años (estudio transversal) pertenecientes a 7 poblaciones ( 2 criollas, 3 matacas, 1 mocovi y 1 toba). Las técnicas de medición se basaron en guías nacionales. Las referencias utilizadas fueron: 1. Centers for Disease Control and Prevention (CDC) (>Pc85: sobrepeso; > Pc95: obesidad); 2. International Obesity Task Force (IOTF) (valores límite sexo-edad específicos). La prevalencia total de sobrepeso, considerando el conjunto de poblaciones, es mayor al aplicar la referencia del CDC en las mujeres, mientras que es levemente superior con IOTF en los varones. Por su parte, la prevalencia total de obesidad, considerando el conjunto de poblaciones, es igual con ambas referencias, en ambos sexos. La mayor parte de las unidades poblacionales evaluadas presentan sobrepeso, aunque la obesidad no parece ser, aún, un problema de salud. Las poblaciones más afectadas son las de Pozo de Maza, la de Criollos de Fortín Belgrano y la de Tobas de Vaca Perdida.
\end{abstract}

$<$ Niños $><$ Adolescentes $><$ Antropometría $><$ Sobrepeso $><$ Obesidad $><$ Índice de Masa Corporal $>$

\begin{abstract}
The evaluation of child nutritional status is highly dependent on the growth charts used. The aim of this study was to compare different references assessing overweight and obesity in children population from Region Chaqueña, through the Body Mass Index. A total of 299, aged 6-15 years, were included (cross-sectional study). The sample was collected in 7 populations (2 criollas, 3 matacas, 1 mocovi and 1 toba). The measurements techniques were based on national guidelines. We compared two references: (1) Centers for Disease Control and Prevention (CDC) (>Pc85: overweight; >Pc95: obesity; (2) International Obesity Task Force (IOTF) (sex-age-specific
\end{abstract}


Padula y Salceda. Sobrepeso y obesidad en niños y adolescentes de la región chaqueña

body mass index cut-offs). The total prevalence of overweight was higher with CDC application respect to the IOTF in woman and in men the relation was reverse. The total prevalence of obesity was identical between references, in both sexes. All populations had a greater prevalence of overweight, but not obesity. The most affected population was Criollos and Wichí from Pozo de Maza, Criollos from Fortín Belgrano and Tobas from Vaca Perdida.

\section{$<$ Children $><$ Anthropometry $><$ Overweight $><$ Obesity $><$ Body Mass Index $>$}

\section{Introducción}

La obesidad es el resultado de un exceso de grasa corporal para la edad, depositada de forma subcutánea o internamente, predominantemente intra-abdominal (Poskitt, 1998). Comenzó a aparecer en el mundo como un problema sanitario de magnitud tanto que la Asamblea Mundial de la Salud, del año 1998, la declaró como una pandemia mundial e inició severas medidas en su contra (O’Donnell, 1999). Este aumento se observa aún en países en desarrollo, los cuales experimentan tradicionalmente altos porcentajes de desnutrición (OMS, 1997; Popkin y Doak, 1998; Juiz et al., 2002). En Argentina se carece de suficiente información sobre la prevalencia global de sobrepeso y obesidad en niños y adolescentes, sobre todo en poblaciones del interior del país (Bejarano et al., 2005; Padula y Salceda, 2009).

En el mundo en desarrollo, puede ser vista como el resultado de una serie de cambios en la dieta, la actividad física, la salud y la nutrición, conocidos conjuntamente como "transición nutricional" (Hoffman et al., 2000). A medida que los países pobres se vuelven más prósperos, adquieren algunos beneficios junto con ciertos problemas de los países industrializados, incluida la obesidad. Se produce un incremento en la importación de alimentos de países industrializados. Como resultado, la dieta tradicional de granos y vegetales, da paso a comidas ricas en grasas y azúcares (Popkin, 1994; Uauy et al., 2000; CDC, 2004).

Es importante remarcar que la obesidad se asocia con factores de riesgo severos para enfermedades cardíacas y otras, tales como hiperlipidemia, hiperinsulinemia, hipertensión y asterosclerosis temprana. Estos factores pueden operar a través de la asociación entre obesidad infantil y adulta, o independientemente de ésta (Cole et al., 2000; Juiz et al., 2002; CDC, 2004). Por tales motivos, el monitoreo de la obesidad en los niños es de suma relevancia.

En epidemiología, el diagnóstico ideal basado en el porcentaje de grasa del cuerpo, es impracticable. De este modo el Índice de Masa Corporal (IMC), aunque menos sensible que los pliegues cutáneos, es muy utilizado (Cole et al., 2000). Refleja la corpulencia y, según muchos investigadores, es un indicador de adiposidad por su alta correlación con la grasa corporal y su independencia con la talla, sobre todo en la infancia media. Se observa una semejanza en las curvas de este índice con las obtenidas para los pliegues cutáneos (Waterlow, 1998). 
En adultos el IMC ha sido ampliamente utilizado para determinar prevalencia de obesidad (Bray et al., 1998; OMS, 1998). En niños y adolescentes la evidencia para utilizar el IMC como indicador de sobrepeso y obesidad se está incrementando rápidamente (Cole et al., 2000; OMS, 1998; Dietz y Robinson, 1998; Kuczmarski et al., 2000). En este sentido, el Centers for Desease Control and Prevention (CDC, 2008), la American Academy of Pediatrics (AAP, 2008) y la Organización Mundial de la Salud (OMS, 2006), recomiendan el uso del IMC para evaluar la prevalencia de sobrepeso y obesidad en niños y adolescentes de entre 2 y 19 años (de Onis et al., 2006; CDC, 2008).

Sin embargo, a causa de que la corpulencia de los niños varía en los diferentes períodos del crecimiento no es posible utilizar el IMC de forma similar que en los adultos, en los que es práctica habitual considerar que un índice de más de 25 puede ser un criterio de sobrepeso y un valor de más de 30 de obesidad (OMS, 1995). En niños y adolescentes, el IMC cambia sustancialmente con la edad (Rolland-Cachera et al., 1982; Cole et al., 1995), por tal motivo, es recomendable el uso de curvas que tomen en consideración la edad y el sexo. En este sentido, el Centers for Disease Control and Prevention (CDC) confeccionó curvas para los Estados Unidos de Norteamérica (Kuczmarski et al., 2000). Otros autores han hecho notar que este patrón no es aplicable a todos los países del mundo, debido a diferencias en la composición corporal y en las dimensiones de los segmentos corporales, en la maduración biológica y en distintas prevalencias de bajo peso de nacimiento, tasas de amamantamiento y enfermedades que habitualmente se asocian con obesidad (O’Donnell y Grippo, 2003). Así, debido a que las mismas carecen de universalidad para realizar comparaciones internacionales, la International Obesity Task Force (IOTF) realizó nuevas curvas (Cole et al., 2000). Obtuvieron datos del IMC de niños de seis naciones, con encuestas representativas de corte transversal del crecimiento en Brasil (1989), Gran Bretaña (1978-93), Hong Kong (1993), Holanda (1980), Singapur (1993) y Estados Unidos de Norteamérica (1963-80). Confeccionaron las curvas para IMC de cada muestra y luego extrapolaron en las mismas los valores límite establecidos para adultos, $25 \mathrm{~kg} / \mathrm{m}^{2}$ para sobrepeso y $30 \mathrm{~kg} / \mathrm{m}^{2}$ para obesidad.

El objetivo de esta investigación es comparar la prevalencia de sobrepeso y obesidad estimada con dos referencias internacionales, en niños y adolescentes de diversas unidades poblacionales aborígenes y criollas de Formosa y Chaco, siguiendo el abordaje teórico-metodológico propuesto en trabajos publicados previamente (Salceda y Tobisch, 2002; Tobisch et al., 2002; Padula et al., 2003; Padula y Salceda, 2004).

\section{Materiales y métodos}

\section{La muestra}

Se registraron 299 niños y adolescentes, 162 mujeres y 137 varones, de edades comprendidas entre los 6 y 15 años de vida (Tabla 1). Los mismos pertenecen a 7 unidades poblacionales, 5 aborígenes y 2 criollas, que habitan actualmente la región del Gran Chaco. A continuación se realiza una breve reseña de cada una de ellas, 
Muestra Nacilamolek: esta unidad representa dentro del esquema tradicional étnico a la población de aborígenes Tobas. Se los conoce con otros nombres tales como Tobas de Sombrero Negro o Tobas-Pilagás. Algunos autores los consideran Pilagás que se autodenominan Tobas (Dell Arcíprete, 1990-1991; Braunstein, 1991-1992). La muestra fue tomada en el año 2001 en el paraje Vaca Perdida, situado en el extremo noroeste de la provincia de Formosa (Tobas VP).

Muestra Wichí de Pozo de Maza: también denominados Matacos, proceden de Wichí montaraces del noroeste de la provincia de Formosa. Los datos fueron recolectados en el año 2001 en la localidad de Pozo de Maza (Wichí PM).

Muestra Wichí de Misión Pozo Yacaré: la muestra proviene de una población de Matacos localizados en la frontera tripartita entre las provincias de Chaco, Formosa y Salta. Los datos fueron recolectados en el año 2001 en el paraje Misión Pozo Yacaré, sobre el curso activo del Río Bermejo (Wichí PY).

Muestra Wichí de Laguna Yema: conformada por individuos Wichí montaraces del centro oeste de la provincia de Formosa. Los datos fueron recolectados en el año 2005 en la localidad de Laguna Yema, Departamento Bermejo de la Provincia de Formosa (Wichí LY).

Muestra Mocoví de Charata: los datos se relevaron en el año 2002 en una población de aborígenes Mocoví de las colonias Juan Larrea y General Necochea de la ciudad de Charata, provincia de Chaco (Mocoví Ch).

Muestra de criollos de Pozo de Maza: la muestra tomada en el año 2001 proviene del noroeste formoseño, de la localidad de Pozo de Maza (Cri PM).

Muestra de criollos de Fortín Belgrano: la muestra fue tomada en el año 2001 entre criollos del paraje Fortín Belgrano, ubicado en la frontera tripartita entre las provincias de Chaco, Formosa y Salta (Cri FB).

Todas las muestras fueron registradas por el equipo multidisciplinario de investigación del Museo de La Plata en el marco de los proyectos "Estudio Antropológico de la Región Meridional del Gran Chaco" (UNLP, 11/N328. CONICET, PIP No. 02812) y "De las Historias étnicas a la Prehistoria, en el Gran Chaco" (CONICET). El afán por coadyuvar en la reconstrucción histórica de la estructura biológica de las poblaciones humanas del Gran Chaco Meridional, incentivó el desarrollo de varios trabajos de campo (1999, 2000, 2001, 2002 y 2005), tendientes a recolectar información antropométrica para el estudio de las similitudes o diferencias somáticas entre poblaciones aborígenes y criollas, con el propósito de llegar a conocer las posibles relaciones entre unas y otras, siempre tratando de reconstruir el origen, diversificación y extensión de los grupos humanos. 
Tabla 1. Estructura etaria de cada una de las poblaciones registradas, por sexo.

\begin{tabular}{|c|c|c|c|c|c|c|c|c|c|c|c|c|c|c|}
\hline \multirow[b]{2}{*}{ Edad } & \multicolumn{2}{|c|}{ Toba VP } & \multicolumn{2}{|c|}{ Wichí PM } & \multicolumn{2}{|c|}{ Wichí PY } & \multicolumn{2}{|c|}{ Wichí LY } & \multicolumn{2}{|c|}{$\begin{array}{c}\text { Mocoví } \\
\text { Ch }\end{array}$} & \multicolumn{2}{|c|}{ Cri PM } & \multicolumn{2}{|c|}{ Cri FB } \\
\hline & fem. & masc. & fem. & masc. & fem. & masc. & fem. & masc. & fem. & masc. & fem. & masc. & fem. & masc. \\
\hline 6 & 0 & 3 & 6 & 0 & 2 & 0 & 3 & 3 & 3 & 2 & 3 & 0 & 0 & 0 \\
\hline 7 & 4 & 6 & 2 & 3 & 4 & 3 & 10 & 3 & 2 & 3 & 0 & 0 & 2 & 0 \\
\hline 8 & 4 & 3 & 4 & 3 & 0 & 2 & 8 & 3 & 0 & 2 & 0 & 0 & 0 & 0 \\
\hline 9 & 3 & 0 & 3 & 4 & 4 & 3 & 7 & 6 & 3 & 2 & 0 & 2 & 2 & 2 \\
\hline 10 & 4 & 5 & 2 & 5 & 0 & 0 & 7 & 5 & 3 & 3 & 2 & 4 & 2 & 0 \\
\hline 11 & 0 & 3 & 2 & 0 & 2 & 2 & 10 & 7 & 3 & 0 & 2 & 0 & 3 & 0 \\
\hline 12 & 0 & 2 & 3 & 0 & 3 & 3 & 6 & 5 & 0 & 2 & 0 & 2 & 0 & 2 \\
\hline 13 & 6 & 0 & 5 & 0 & 0 & 0 & 7 & 9 & 0 & 4 & 3 & 3 & 0 & 2 \\
\hline 14 & 0 & 0 & 0 & 0 & 0 & 0 & 2 & 0 & 0 & 0 & 0 & 0 & 0 & 0 \\
\hline 15 & 2 & 0 & 2 & 7 & 0 & 0 & 0 & 4 & 0 & 0 & 2 & 0 & 0 & 0 \\
\hline $\begin{array}{c}\text { Total por } \\
\text { sexo }\end{array}$ & 23 & 22 & 29 & 22 & 15 & 13 & 60 & 45 & 14 & 18 & 12 & 11 & 9 & 6 \\
\hline Total & & 45 & & 51 & & 28 & 10 & 05 & & 32 & & 23 & 1 & 15 \\
\hline
\end{tabular}

\section{Los datos}

Variables antropométricas: 1) Edad (E), se registró en meses y se establecieron las categorías de edad según el criterio de edad centrada (Jordán, 1988). 2) Peso (P), se utilizó la balanza de pie, con graduaciones cada 100 gramos. El registro se realizó en kilogramos y gramos como fracción decimal. Los niños fueron pesados descalzos y con ropa, descontándose luego el peso de la misma. 3) Talla (T), se usó un antropómetro de metal y se registró en metros. Las mediciones se efectuaron con el niño descalzo. Las técnicas utilizadas para la toma de mediciones antropométricas se basaron en las Guias de crecimiento y desarrollo de la Sociedad Argentina de Pediatría (2001). Todos los datos fueron registrados por una única persona (GP), previamente entrenada y según protocolos estandarizados.

Se estableció el IMC, peso/talla ${ }^{2}$ según la fórmula $\mathrm{kg} / \mathrm{m}^{2}$, y para determinar la prevalencia de sobrepeso y obesidad, se utilizaron dos referencias internacionales diferentes: 1- Center for Disease Control and Prevention (CDC, 2000) de Estados Unidos de Norteamérica (Kuczmarski et al., 2000), con los valores límite recomendados por el US Expert Committee on Childhood Obesity (Barlow y Dietz, 1998), Pc85 para determinar sobrepeso y Pc95 para obesidad. 2- International Obesity Task Force (IOTF, 2000), con los valores límite sexo-edad específicos, publicados en el estudio (Cole et al., 2000).

Si bien fueron registrados todos los niños en cada una de las unidades poblacionales, la casuística resultó muy baja. Tal situación motivó un nuevo agrupamiento de los niños dentro de cada unidad poblacional, a los fines comparativos, considerando dos categorías 
de edad y teniendo como criterio de corte la edad a la que, por lo menos las niñas, podrían comenzar con la aceleración pubertal del crecimiento: a) 6 a 10 años; b) 11 a 15 años. Para la comparación de las frecuencias porcentuales entre referencias, por sexo y categoría etaria, se utilizó la Prueba $\chi^{2}$ de Pearson, a través del empleo del programa Epi Info 6.0 (CDC/OMS, 2001) a un nivel de confianza del 95\%.

\section{Resultados}

La prevalencia total de sobrepeso, considerando el conjunto de poblaciones, es mayor al aplicar la referencia del CDC en las mujeres y levemente superior con IOTF en los varones. Al discriminar por unidad poblacional, se observan diferencias en la estimación de la prevalencia de sobrepeso sólo para las poblaciones Wichi PM y Wichi LY, en ambos sexos. En las mujeres la prevalencia es superior con CDC para la población Wichi PM en ambas categorías de edad (menor de 10 años $=18,34 \%$ vs. $28,34 \%$; mayor de 11 años $=14 \%$ vs. $28 \%)$ y sólo en los mayores de 11 años para los Wichi LY $(2,86 \%$ vs. $6,2 \%)$. En los varones se observa una mayor prevalencia con IOTF para la población Wichí PM en los menores de 10 años (30,34\% vs. 26,34\%), mientras que en la población Wichi LY el porcentaje es superior con CDC para este mismo grupo de edad $(0 \%$ vs. $6,66 \%$ ) (Tabla 2).

En el caso de las mujeres se observa, de manera general y sin discriminar por referencias, que las poblaciones Wichi PM y Tobas VP son las más afectadas por sobrepeso. En general, son los menores de 10 los que presentan las prevalencias más altas, con ambas referencias. En el caso de los varones, las poblaciones Wichi PM y Criollos FB son las más afectadas, mientras que en las poblaciones Criollos PM, Wichi PY y Mocovi Ch, las frecuencias son nulas. Al igual que en las mujeres son los menores de 10 años los que presentan los valores más altos para sobrepeso, con ambas referencias (Tablas 2).

Por su parte, la prevalencia total de obesidad, considerando el conjunto de poblaciones, es igual con ambas referencias tanto en mujeres como en varones. Asimismo, al discriminar por unidad poblacional, se observan frecuencias idénticas de individuos con obesidad entre ambas referencias, para ambos sexos y a todas las edades (Tabla 3).

En el caso de las mujeres se aprecia, de manera general y sin discriminar por referencias, que las únicas poblaciones afectadas son las de Pozo de Masa, tanto los Criollos como los Wichí, para el resto de las poblaciones la obesidad no representa un problema de salud importante. En general, las mujeres mayores de 11 años presentan un porcentaje levemente superior de obesidad que las menores de 10 años, con ambas referencias. En el caso de los varones, las únicas poblaciones afectadas son Criollos FB y Tobas VP, nuevamente para el resto de las poblaciones la obesidad no representa un problema de salud. En general, son los niños menores de 10 años quienes exhiben la mayor prevalencia de obesidad, con ambas referencias (Tabla 3). 
Folia Histórica del Nordeste, № 18 (Resistencia, 2010) IIGHI, CONICET - IH, UNNE

Tabla 2. Sobrepeso: frecuencias porcentuales por sexo, referencia y categoría etaria.

\begin{tabular}{|l|r|r|r|r|r|r|r|r|}
\hline \multirow{2}{*}{ Sobrepeso } & \multicolumn{4}{|c|}{ Mujeres } & \multicolumn{3}{|c|}{ Varones } \\
\cline { 2 - 10 } & \multicolumn{2}{|c|}{ IOTF } & \multicolumn{2}{|c|}{ CDC } & \multicolumn{2}{|c|}{ IOTF } & \multicolumn{2}{c|}{ CDC } \\
\hline Población & $\leq \mathbf{1 0}$ & $\mathbf{1 1}$ & $\leq \mathbf{1 0}$ & $\mathbf{1 1}$ & $\leq \mathbf{1 0}$ & $\geq \mathbf{1 1}$ & $\leq \mathbf{1 0}$ & $\geq \mathbf{1 1}$ \\
\hline Criollos PM & 0 & 10 & 0 & 10 & 0 & 0 & 0 & 0 \\
\hline Wichí PM & 18,34 & 14 & 28,34 & 28 & 30,34 & 8,58 & 26,34 & 8,58 \\
\hline Criollos FB & 0 & 6,66 & 0 & 6,66 & 0 & 20 & 0 & 20 \\
\hline Wichí PY & 0 & 0 & 0 & 0 & 0 & 0 & 0 & 0 \\
\hline Tobas VP & 15 & 6,66 & 15 & 6,66 & 8 & 0 & 8 & 0 \\
\hline Mocoví Ch & 10 & 0 & 10 & 0 & 0 & 0 & 0 & 0 \\
\hline Wichí LY & 8,86 & 2,86 & 4,86 & 6,2 & 0 & 4 & 6,66 & 4 \\
\hline Total & 7,46 & 5,74 & 8,31 & 8,22 & 5,48 & 4,65 & 5,86 & 4,65 \\
\hline
\end{tabular}

Tabla 3. Obesidad: frecuencias porcentuales por sexo, referencia y categoría etaria.

\begin{tabular}{|l|r|r|r|r|r|r|r|r|}
\hline \multirow{2}{*}{ obesidad } & \multicolumn{4}{|c|}{ Mujeres } & \multicolumn{3}{|c|}{ Varones } \\
\cline { 2 - 9 } & \multicolumn{2}{|c|}{ IOTF } & \multicolumn{2}{|c|}{ CDC } & \multicolumn{2}{|c|}{ IOTF } & \multicolumn{2}{|c|}{ CDC } \\
\hline Población & $\leq \mathbf{1 0}$ & $\mathbf{1 1}$ & $\leq \mathbf{1 0}$ & $\mathbf{1 1}$ & $\leq \mathbf{1 0}$ & $\mathbf{1 1}$ & $\leq \mathbf{1 0}$ & $\geq \mathbf{1 1}$ \\
\hline Criollos PM & 0 & 10 & 0 & 10 & 0 & 0 & 0 & 0 \\
\hline Wichí PM & 8 & 0 & 8 & 0 & 0 & 0 & 0 & 0 \\
\hline Criollos FB & 0 & 0 & 0 & 0 & 10 & 0 & 10 & 0 \\
\hline Wichí PY & 0 & 0 & 0 & 0 & 0 & 0 & 0 & 0 \\
\hline Tobas VP & 0 & 0 & 0 & 0 & 0 & 6,66 & 0 & 6,66 \\
\hline Mocoví Ch & 0 & 0 & 0 & 0 & 0 & 0 & 0 & 0 \\
\hline Wichí LY & 0 & 0 & 0 & 0 & 0 & 0 & 0 & 0 \\
\hline Total & 1,14 & 1,43 & 1,14 & 1,43 & 1,43 & 0,94 & 1,43 & 0,94 \\
\hline
\end{tabular}

\section{Discusión}

Hoy en día existe una gran confusión en relación a la evaluación del sobrepeso y la obesidad, debido a la ausencia de consistencia y coincidencia en los criterios de clasificación y en la elección de las poblaciones de referencia y valores límite, tornando muy difícil la comparación de los datos (Flegal et al., 2001; Bejarano et al., 2005; Sweeting, 2007). 
Padula y Salceda. Sobrepeso y obesidad en niños y adolescentes de la región chaqueña

En Argentina la Subcomisión de Epidemiología y el Comité de Nutrición de la Sociedad Argentina de Pediatría han publicado el "Consenso sobre factores de riesgo de enfermedad cardiovascular en pediatría. Obesidad" (SAP, 2005). Y, aunque en el mismo se aconseja el uso del P//T como indicador de sobrepeso y obesidad en niños menores de 6 años a nivel epidemiológico, recientemente la OMS publicó las nuevas Curvas de Crecimiento Infantil (OMS, 2006) donde se recomienda el uso del IMC para estimar las prevalencias de sobrepeso y obesidad en niños y adolescentes de entre 2 y 19 años.

Se ha hecho notar que cuando se evalúa una misma población con la referencia IOTF y con la del CDC surgen diferencias importantes en la estimación de sobrepeso y obesidad, especialmente en la adolescencia y en la niñez temprana (más de 10\%) (O’Donnel y Grippo, 2003). En nuestro trabajo las frecuencias son diferentes para sobrepeso sólo para las unidades poblacionales Wichí PM y Wichí LY. En las mujeres de la población Wichi PM y varones Wichi LY las frecuencias son superiores con CDC, mientras que en los varones Wichi PM y mujeres Wichi LY se da la situación inversa. Estas diferencias no superan las reportadas por O'Donnell y Grippo (2003) y no son estadísticamente significativas. La prevalencia de obesidad es exactamente igual con ambas referencias. Por su parte, en un estudio previo realizado en una muestra de niños del Gran La Plata (Buenos Aires, Argentina), observamos que mientras la prevalencia de sobrepeso es similar con la aplicación de ambas tablas, la prevalencia de obesidad es significativamente menor al aplicar las tablas del IOTF, con una diferencia máxima de $11,1 \%$ en mujeres y de $14,5 \%$ en varones, ambos a la edad de 4 años (Padula y Salceda, 2008). Otros autores encontraron resultados semejantes, donde la referencia IOTF tiende a subestimar la obesidad (Chinn y Rona, 2001; Wang y Wang, 2002; Bejarano et al., 2005; Serra-Majem et al., 2007). Flegal y colaboradores (2001) compararon la prevalencia de obesidad en niños y adolescentes de Estados Unidos a partir de los datos de las NHANES $(1995,1999)$ utilizando los criterios del CDC y IOTF. Obtuvieron resultados similares, con porcentajes superiores con CDC en los niños más pequeños y mayores con IOTF en los más grandes. Los autores atribuyeron las diferencias a la disparidad de los datos, de los métodos de suavizamiento, del agrupamiento etario y del encuadre teórico.

Debe hacerse una serie de consideraciones en referencia a las curvas aquí comparadas. La referencia CDC está basada sólo en población estadounidense, mientras que la referencia IOTF incluyó poblaciones de diversas partes del mundo aunque, según palabras del mismo Cole, la población con la que se construyeron las curvas de la IOTF es menos que ideal "la cuál probablemente refleje adecuadamente a las poblaciones del oeste, pero no representa al resto del mundo". La referencia del CDC utiliza valores límite con base estadística para determinar la prevalencia de sobrepeso y obesidad, en contraste, la de IOTF considera valores que no están relacionados con la verdadera distribución de la población de referencia. De hecho, los valores límites son aquellos extrapolados de 25 y $30 \mathrm{Kg} / \mathrm{m}^{2}$ a la edad de 18 años. Respecto a este punto, Really et al. (2000) publicaron un trabajo cuyo objetivo era determinar la sensibilidad y especificidad de los valores límite en la determinación de la prevalencia de obesidad. Tomando el Pc95, con la referencia británica (UK 1900) y aquel recomendado por la IOTF para la edad de 7 años, concluyeron 
que hay una disminución de la sensibilidad utilizando el criterio de la IOTF. Concluyeron que la prevalencia de obesidad en estudios epidemiológicos queda substancialmente subestimada utilizando los valores de la IOTF. Otros autores confirman estos resultados (Kain et al., 2002).

Por otra parte, en la literatura se ha reseñado que el sobrepeso tiene una mayor incidencia en las niñas respecto de los niños (O'Donnell, 1999). En nuestro trabajo, considerando las frecuencias totales de sobrepeso, las mujeres están más afectadas que los varones tanto en menores de 10 años como en mayores y con ambas referencias. En el caso de la obesidad, se observa también una mayor prevalencia en las mujeres mayores de 11 años y un porcentaje levemente superior en los varones menores de 10 años, con ambas referencias. El porcentaje total de niñas afectadas por sobrepeso y obesidad es levemente superior al de niños ( 11,11 vs $10,81 \%$ y 1,85 vs $1,45 \%$, respectivamente). Esta situación se observa en Criollos PM, Tobas VP, Mocoví Ch y Wichí LY, mientras que en las poblaciones Wichí PM y Criollos FB, los varones presentan frecuencias mayores para sobrepeso que las mujeres. Kromeyer y col. (1999), así como César y col. (1990), informaron mayor número de mujeres con sobrepeso y obesidad, en tanto que Kain y col (2002) observaron un ligero predominio de obesidad en el sexo femenino, al igual que Dei-Cas y copartícipes (2002). Por su parte, los datos publicados por Átala y col. (2001) mostraron que la obesidad no se asoció con el sexo.

En la Argentina, Agrelo y colaboradores (1986) en la ciudad de Córdoba encontraron una prevalencia de obesidad del 6,3\%, cifra superior a la obtenida en nuestra investigación $(1,66 \%)$. En estudiantes secundarios de la ciudad de Corrientes se detectó una prevalencia de sobrepeso y obesidad del $10,9 \%$ y 2,2\%, respectivamente (Martínez et al., 2001), valores semejantes a los hallados en nuestro estudio. Bazán (2001) estimó una prevalencia de obesidad del 13\% al evaluar niños de 6 a 14 años de las provincias de Buenos Aires, Corrientes, La Rioja y San Luis, utilizando el criterio de percentilo 95 del IMC, cifra muy superior a la nuestra. De acuerdo con una investigación llevada a cabo por la Sociedad Argentina Pediatría, el 20,8\% de los encuestados de entre 10 y 19 años, representativos de todo el país, presentaron sobrepeso (Navarra, 2001). En tanto en Chile, distintos trabajos consultados coinciden en afirmar que la prevalencia de estas enfermedades nutricionales ha crecido significativamente en ese país en el último tiempo, con cifras superiores a las obtenidas por nosotros (Ivanovic et al., 1990; Parodi et al., 1993; Rozowski y Arteaga, 1997; Átala et al., 2001).

Estudios realizados en Tierra del Fuego por el Centro de Estudios sobre Nutrición Infantil (CESNI) indican aumento de la obesidad con el paso de la infancia a la adolescencia, principalmente en las clases más humildes (Biasotti, 2000; Agostini, 2000). Sin embargo, en nuestro trabajo son los menores de 10 años, para ambos sexos y referencias, los que presentan una mayor frecuencia global de sobrepeso. Mientras que en el caso de la obesidad se observa una distinción por sexo, las mujeres presentan valores superiores a partir de los 11 años y los varones para los menores de 10 años. En un trabajo realizado por Bejarano y colaboradores (2005), el primero que proporcionó información sobre la tendencia de la prevalencia de sobrepeso y obesidad a lo largo de 6 años, se 
observó también un porcentaje superior de sobrepeso en los niños menores de 10 años, tanto en mujeres como en varones, asimismo, en el caso de la obesidad se observa una diferenciación por sexo, aunque inversa a la planteada en este trabajo.

Actualmente, se cree que las diferencias entre poblaciones son fundamentalmente socioambientales más que genéticas (Bustos et al., 2001). Así, los niños sanos de alrededor del mundo que son criados en un ambiente saludable, siguiendo las prácticas alimentarias recomendadas, tienen un patrón de crecimiento similar (de Onis et al., 2006). El abordaje teórico-metodológico propuesto en este trabajo, y ya explicado en estudios previos (Salceda y Tobisch, 2002; Tobisch et al., 2002; Padula et al., 2003; Padula y Salceda, 2004), según el cuál las muestras son consideradas como unidades poblacionales distintas a través de criterios etnográficos, lingüísticos y geográficos y no agrupadas según su nominación vulgar, confirman esta observación. Ya que, en general, existen más semejanzas a nivel nutricional entre poblaciones que comparten un mismo hábitat (ej. Wichí de Pozo de Maza con Criollos de Pozo de Maza) que entre aquellas que se adscriben clásicamente a un determinado grupo étnico (ej.Wichí Pozo de Maza con Wichí Laguna Yema).

Es curioso que las poblaciones más afectadas por sobrepeso y obesidad, tales como Wichí PM, Criollos PM, Criollos FB y Tobas VP, son aquellas en las que habíamos encontrado anteriormente los porcentajes más altos de individuos distróficos por déficit (Padula et al., 2003). Así, en estas unidades poblacionales conviven ambos extremos de la malnutrición, confirmándose la existencia del paradigma nutricional emergente “obesidad en la pobreza” (OPS, 1997; Peña y Bacallao, 2001; Bejarano et al., 2005). Por su parte, los Mocoví y los Wichí de Misión Pozo Yacaré son los que experimentan los menores porcentajes de sobrepeso (llegando a ser nulos en el caso de los varones) y obesidad (inexistentes para ambos sexos) y presentan, asimismo, las menores prevalencias de individuos distróficos por déficit (Padula et al., 2003). La población Mocoví se ha incorporado a la vida urbana de la ciudad de Charata, mientras que la Wichí de Pozo Yacaré es, de todas las poblaciones analizadas, la que mantiene con mayor fuerza las pautas de subsistencia tradicionales, siendo muy importante el componente pescador.

Si bien tradicionalmente los aborígenes del Gran Chaco han sido cazadoresrecolectores-pescadores nómades o semi-nómades, hoy día se circunscriben a parajes o localidades pequeñas (Valeggia et al., 2002; Padula et al., 2003; Padula y Salceda, 2006), observándose una gran dependencia a la escasa ayuda provista por el Estado. En general, los niños comen en la escuela llevando parte de la comida a su hogar. La dieta suele ser rica en hidratos de carbono y pobre en proteínas, base de los problemas nutricionales consignados. Así, estos cambios observados en la dieta, la actividad física, la salud y la subsistencia, son los artífices de los problemas nutricionales observados.

\section{Conclusión}

Para la estimación de la prevalencia de sobrepeso las diferencias entre referencias son muy bajas, mientras que para la obesidad son inexistentes. La mayor parte de las unidades poblacionales evaluadas presentan sobrepeso, aunque la obesidad no parece ser, 
aún, un problema de salud. Las poblaciones más afectadas son las de Pozo de Maza, tanto Wichis como Criollos, la de Criollos de Fortín Belgrano y la de Tobas de Vaca Perdida.

\section{Referencias Bibliográficas}

Agrelo F, Lobo B, Bazán N, Cinman N, Villafañe L, Actis C, Rodríguez A 1986. Prevalencia de obesidad en un grupo de escolares de bajo nivel socioeconómico. Arch Argent Pediatr 84:512.

Átala E, Urteaga C, Rebolledo A, Delfín S, Ramos R (2001). Prevalencia de obesidad en escolares de la Región de Aysén. Arch Argent Pediatr 99(1):28-33.

Barlow S, Dietz W (1998). Obesity evaluation and treatment: expert committee recommendations. Pediatrics 102:E29-36.

Bejarano I, Dipierri J, Alfaro E, Quispe Y y Cabrera G (2005). Evolución de la prevalencia de sobrepeso, obesidad y desnutrición en escolares de San Salvador de Jujuy. Arch Argent Pediatr 103(2)/0 101.

Braunstein J (1991-1992). “Presentación”. En: Hacia una Nueva Carta Étnica del Gran Chaco II. Informe de avance. Centro del Hombre Antiguo Chaqueño (Chaco). Las Lomitas, Formosa. $1-8$.

Bray GA, Bouchard C, James WPT (1998). Handbook of Obesity. Dekker M, editor. New York.

Bustos P, Amigo H, Muñoz SR, Martorell R (2001). Growth in indigenous and nonindigenous Chilean schoolchildren from 3 poverty strata. Am J Public Health 91:1645-9.

Centers for Disease Crontrol and Prevention 2004. "The nutrition transition and obesity.

Centers for Disease Crontrol and Prevention. About BMI for Children and Teen”. [En línea] 2008 junio 20 .

http://www.cdc.gov/nccdphp/dnpa/healthyweight/assessing/bmi/childrens_BMI/about_childrens BMI.htm.

César M, Raizman H, Tonietti M (1990). Análisis de las características de 823 niños que consultaron por obesidad a un Servicio de Nutrición Infantil. Publicación Carpt 87-9.

Chinn S, Rona RJ (2001). Can the increase in body mass index explain the rising trend in asthma in children?. Thorax 56:845-50.

Cole TJ, Freeman JV, Preece MA (1995). Body mass index reference curves for the UK, 1990. Arch Dis Child 73:259.

Cole TJ, Bellizzi MC, Flegal KM y Dietz WH (2000). Survey Overweight and Obesity Worldwide: International Establishing a standard definition for child. BMJ 320: 1240-1243.

Dei-Cas PG, Dei-Cas SA y Dei-Cas IJ (2002). Sobrepeso y obesidad en la niñez. Relación con factores de riesgo. Arch Argent Pediatr 100(5) / 368.

Dell'Arcíprete A (1990-1991). Lugares de los Pilagás. En: Hacia una Nueva Carta Étnica del Gran Chaco II. Informe de avance. Centro del Hombre Antiguo Chaqueño (Chaco). Las Lomitas, Formosa. 58-85.

De Onis M, Onyango AW, Borghi E, Garza C, Yang H (2006), for the WHO Multicentre Growth Reference Study Group. Comparison of the World Health Organization (WHO) Child Growth Standards and the National Center for Health Statistics/WHO international growth reference: implications for child health programmes. Public Health Nutrition 9(7):942-7.

Dietz WH, Robinson TN (1998). Use of the body mass index (BMI) as a measure of overweight in children and adolescents. J Pediatr 132:191-3.

Epidat (2003). Programa para análisis epidemiológico de datos tabulados. Versión 3.0. Xunta de Galicia. OPS / OMS. 
Padula y Salceda. Sobrepeso y obesidad en niños y adolescentes de la región chaqueña

Epi Info 6. (2001). Procesador de texto, Base de datos y Estadística, para la Salud Pública. Versión 6.04d. CDC / OMS.

Flegal KM, Ogen C, Wei R, Kuczmarski RL, Johnson C (2001). Prevalence of overweight in US children: comparison of US growth charts from the Center for Disease Control and Prevention with other reference values for body mass index. Am J Clin Nutr 73(6):1086-1093.

Hoffman DJ, Sawaya AL, Verreschi I, Tucker KL y Roberts SB (2000). Why are nutritionally stunted children at increased risk of obesity? Studies of metabolic rate and fat oxidation in shantytown children from São Paulo, Brazil1-3. Am J Clin Nutr 72:702-707.

Ivanovic R, Olivares M, Ivanovic D (1990). Estado nutricional en escolares chilenos urbanos y rurales de la Región Metropolitana, 1986-1987. Rev Chil Pediatr 61(4):210-7.

Jordán JR (1988). El lactante de 0 a 2 años: Antropometría y Crecimiento. En: Crecimiento y Desarrollo. Hechos y tendencias. Cusminsky M, Moreno E y Suárez Ojeda EN (eds.). 184209.

Juiz CI, Montagna MG, Salazar R, Gotthelf S y Tríboli Pisi F (2002). Perfil familiar, antropométrico y humoral de niños obesos de la ciudad de Salta. Arch Argent Pediatr 100(?) / 1.

Kain J, Albala C, García F, Andrade M (1998). Obesidad en el preescolar: evolución antropométrica $y$ determinantes socioeconómicos. Rev Med Chile 126:271-8.

Kromeyer-Hauschild K, Zellner K, Jaeger U, Hoyer H (1999). Prevalence of overweight and obesity among school children in Jena (Germany). Int J Obes 23:1143-50.

Kuczmarski RJ, Ogden CL, Grummer-Strawn LM, Flegal KM, Guo SS, Mei R, et al (2000). CDC growth charts: United States. Advance data from vital and health statistics, no. 314. National Center for Health Statistics; Hyattsville (MD).

O’Donnell A y Carmuega E, coordinadores (1999). Hoy y Mañana. Salud y calidad de vida de la Niñez Argentina. Documentos y discusiones del taller realizado en Villa La Angostura.

O’Donnell AM y Grippo B (2003). Las tablas de crecimiento como patrón de referencia. Una reflexión. Arch Argent Pediatr 101(1): 57-60.

Organización Mundial de la Salud (1995). Physical status: the use and interpretation of anthropometry. Geneva.

Organización Mundial de la Salud (1997). Obesity: preventing and managing the global epidemic. Geneva.

Organización Mundial de la Salud (1998). Obesity: Preventing and Managing the Global Epidemic. Report of a WHO Consultation on Obesity. WHO/NUT/NCD/981. WHO. Geneva.

Organización Panamericana de la Salud (1997). Temas de actualidad. Componentes educativos de los programas para la promoción de la salud escolar. Pan Am J Public Health 2(3):209-214.

Padula G, Salceda SA, Tobisch AC, Porterie AP (2003). Evaluación antropométrica del estado nutricional de niños y adolescentes de la Región del Gran Chaco. Actas del XXIII Encuentro de Geohistoria Regional. Instituto de Investigaciones Geohistóricas (IIGH-CONICET). 339349.

Padula G, Salceda SA (2004). Morfometría aplicada en niños y adolescentes de la Región Chaqueña. Actas del XXIV Encuentro de Geohistoria Regional. Instituto de Investigaciones Geohistóricas (IIGH-CONICET). 426-432.

Padula G, Salceda SA (2006). Laguna Yema: evaluación antropométrica del estado nutricional de niños y adolescentes. Actas del XXVI Encuentro de Geohistoria Regional. Instituto de Investigaciones Geohistóricas (IIGH-CONICET). 369-378.

Padula G, Salceda SA (2008). Comparación entre referencias de las prevalencias de sobrepeso y obesidad, estimadas a través del Índice de Masa Corporal, en niños de Argentina. Arch 
Latinoam Nutr 58(4):330-335.

Peña M, Bacallao J (2001). La obesidad y sus tendencias en la región. Pan Am J Public Health 10(2):75-77.

Popkin BM (1994). The nutrition transition in low-income countries: an emerging crisis. Nutr Rev 285-95.

Popkin BM, Doak CM (1998). The obesity epidemic is a worldwide phenomenon. Nutr Rev 10614.

Poskitt EME (1998). Childhood obesity and growth. En: The Cambridge encyclopedia of human growth and development. Cambridge University Press. Ulijaszek SJ, Johnston FE y Preece, MA (eds.): 332-333.

Reilly JJ, Dorosty AR, Emmett PM and the ALSPAC Study Team (2000). Identification of the obese child: adequacy of the body mass index for clinical practice and epidemiology. Int $\mathrm{J}$ Obes Relat Metab Disord 24:1623-7.

RollandCachera MF, SempÈ M, GuilloudBataille M, Patois E, PequignotGuggenbuhl F, Fautrad V (1982). Adiposity indices in children. Am J Clin Nutr 36:17884.

Rozowski J, Arteaga A (1997). El problema de la obesidad y sus características alarmantes en Chile. Rev Med Chile 125:1217-24.

Salceda SA, Tobisch CA (2002). Diferencias dermatoglificas digitales en poblaciones aborígenes del Chaco Argentino. Actas del XXII Encuentro de Geohistoria Regional, Instituto de Investigaciones Geohistóricas (IIGH-CONICET). 822-829.

Serra-Majem L, Ribas-Barba L, Pérez-Rodrigo C (2007). Methodological limitations in measuring childhood and adolescent obesity and overweight in epidemiological studies: does overweight fare better than obesity?. Public Health Nutr 10(10A):1112-20.

Sociedad Argentina de Pediatría (2001). Guías para la evaluación del crecimiento. $2^{\mathrm{a}}$ ed SAP, Comité de Crecimiento y Desarrollo. Buenos Aires.

Sociedad Argentina de Pediatría (2005). Subcomisión de Epidemiología y Comité de Nutrición. Consenso sobre factores de riesgo de enfermedad cardiovascular en pediatría. Obesidad. Arch Argent Pediatr 103 (3):262-81.

Sweeting HN (2007). Measurement and definitions of obesity in childhood and adolescence: a field guide for the uninitiated. J Nutr 6:32.

Tobisch CA, Salceda SA, García Mancuso R (2002). Frecuencia de diseños dermatoglíficos digitales de cuatro unidades poblacionales de aborígenes chaquenses. Actas del XXII Encuentro de Geohistoria Regional, Instituto de Investigaciones Geohistóricas (IIGH-CONICET). 977-985.

Uauy R, Mize CE, Castillo-Duran C (2000). Fat intake during childhood: metabolic responses and effects on growth1. Am J Clin Nutr 72(suppl):1354S-60S.

Valeggia C, Faulkner KM, Ellison PT (2002). Crecimiento en lactantes de una comunidad Toba de Formosa. Arch Argent Pediatr 100(2): 103-109.

Wang Y, Wang JQ (2002). A comparison of international references for the assessment of child and adolescent overweight and obesity in different populations. Europ J Clin Nutr 56: 973-82.

Waterlow JC (1996). Malnutrición proteico-energética. Organización Panamericana de la Salud. Organización Mundial de la Salud. Publicación Científica Nro. 555. Washington D.C. 